\title{
BOVIDS: A deep learning-based software for pose estimation to evaluate nightly behavior and its application to Common Elands (Tragelaphus oryx) in zoos
}

\author{
Jennifer Gübert ${ }^{1}$, Max Hahn-Klimroth ${ }^{2}$, and Paul W. Dierkes ${ }^{3}$ \\ ${ }^{1}$ Goethe University Frankfurt \\ ${ }^{2} \mathrm{TU}$ Dortmund University \\ ${ }^{3}$ Goethe-Universitat Frankfurt am Main
}

November 18, 2021

\begin{abstract}
Only a few studies on the nocturnal behavior of African ungulates exist so far, with mostly small sample sizes. For a comprehensive understanding of nocturnal behavior, this database needs to be expanded. Zoo animals offer a good opportunity to lay the corresponding foundations. The results can provide clues for the study of wild animals and furthermore contribute to a better understanding of animal welfare and better husbandry conditions in zoos. To tackle this open question, we developed a stand-alone open-source software based on deep learning techniques, named BOVIDS (Behavioral Observations by Videos and Images using a Deep-Learning Software). This software is used to identify ungulates in their enclosure and to determine crucial behavioral poses on video material with an accuracy of $99.4 \%$. A case study on 25 Common Elands (Tragelaphus oryx) out of 5 EAZA zoos with a total of 11,411 hours video material out of 822 nights is conducted, yielding the first detailed description of the nightly behavior of Common Elands. Our results indicate that age and sex are influencing factors on the nocturnal activity budget, the length of behavioral phases as well as the number of phases per behavioral state during the night. Finally, the results suggest the existence of species-specific rhythms that open future research directions.
\end{abstract}

\section{Hosted file}

BOVIDS_submission.pdf available at https://authorea.com/users/389245/articles/545953-bovidsa-deep-learning-based-software-for-pose-estimation-to-evaluate-nightly-behavior-and-itsapplication-to-common-elands-tragelaphus-oryx-in-zoos 